\title{
Communicating threats of future climate change to building design practitioners
}

\author{
M. S. Gul, V. Ingram \& G. F. Menzies \\ School of the Built Environment, Heriot-Watt University, UK
}

\begin{abstract}
This paper describes a communication strategy aiming to bridge the gap between research and practice, by raising awareness of future overheating risks in buildings. It is the outcome of four years of intensive research to design a tool which can identify risk of overheating: the Low Carbon Futures (LCF) tool. A specific workshop, with a targeted audience, was held to understand better how the LCF tool can be adapted and used in industry. Participants included experienced private sector building design professionals, local authorities, housing associations, building services engineering consultancies, and members of corporate organisations. A recurring theme throughout the workshop was that the LCF tool is one that is useful to all sectors, and that further investigation should be made into expanding the use of the tool. The feedback gathered will be used to inform a number of output styles, based on client type.

Keywords: climate change, future overheating risk, practitioner's engagement.
\end{abstract}

\section{Introduction}

As climate change intensifies [1], developments designed using historic climate data may not be able to cope with new climate conditions. For instance, there are significant risks of increases in mean temperature by the end of the century, which may lead to buildings severely overheating in certain parts of the UK [2]. New buildings are now being designed with climate in mind, the extent to which this is considered is usually with respect to making buildings more energy efficient and thus mitigating against climate change [3]. The 2010 release of the UK probabilistic climate data projections (UKCP09) provides in depth information to understand risks posed by an uncertain future [2]. The complexity and form of some of these climate projections can provide a barrier to their use; probabilistic 
climate descriptions for multiple future scenarios are not necessarily compatible with the timescales and resources associated with real building projects.

Any message that warns of the dangers of climate change should also clearly provide solutions - solutions that are both effective, as well as possible for the audience member to perform or support [4]. The Low Carbon Futures (LCF) project developed a methodology $[5,6]$ that integrates these complex probabilistic projections to understand potential overheating risks in the UK. To effectively disseminate the outcomes of the LCF tool to practitioners, the LCF team organised a workshop in London to identify the next steps to facilitate its wider adoption. The outcomes of this workshop are discussed in this paper.

\section{Background}

\subsection{The Low Carbon Futures tool}

The LCF Project, as part of the Adaptation and Resilience to Climate Change (ARCC) programme investigated the use of the UKCP09 climate projections within building performance simulations and developed a methodology to identify the risk of buildings failing in a future climate, due either to excessive overheating or to inadequate capacity in existing cooling systems [7]. The LCF tool combines dynamic building simulation and probabilistic climate projections, both of which are computationally intensive, in a format that is potentially compatible with commercially available software. The LCF tool not only assesses the future overheating risks but also suggests the effect of the adaptation choices, so that the design can be sensitive to the uncertainties of future climate projections. The LCF tool can therefore be applied to the simulation results of any building and use any criterion for overheating or building failure $[5,6]$.

\subsection{Engagement with practitioners}

The breadth of information from climate datasets and detailed building simulation can result in relatively complex output which may not be meaningful to a wide spectrum of building professionals. Figure 1 shows the outcome of the LCF tool in the form of a cumulative probability graph, quantifying and indicating the risk of overheating within a building $[5,6]$. To translate this information into something more concise and specific, a qualitative study was carried out to interact with building practitioners to explore the issues of overheating and how this is being overcome in domestic and non-domestic buildings [8-12]. This ensures the applicability and outcomes of the tool are best suited to meet industry needs in implementing adaptive measures to the buildings, to cope better with the consequences of climate change. Questionnaires, focus groups and semistructured interviews were carried out to examine the general design considerations and to evaluate current practices with regards to the probability of increased summer temperatures in future [8-12]. 


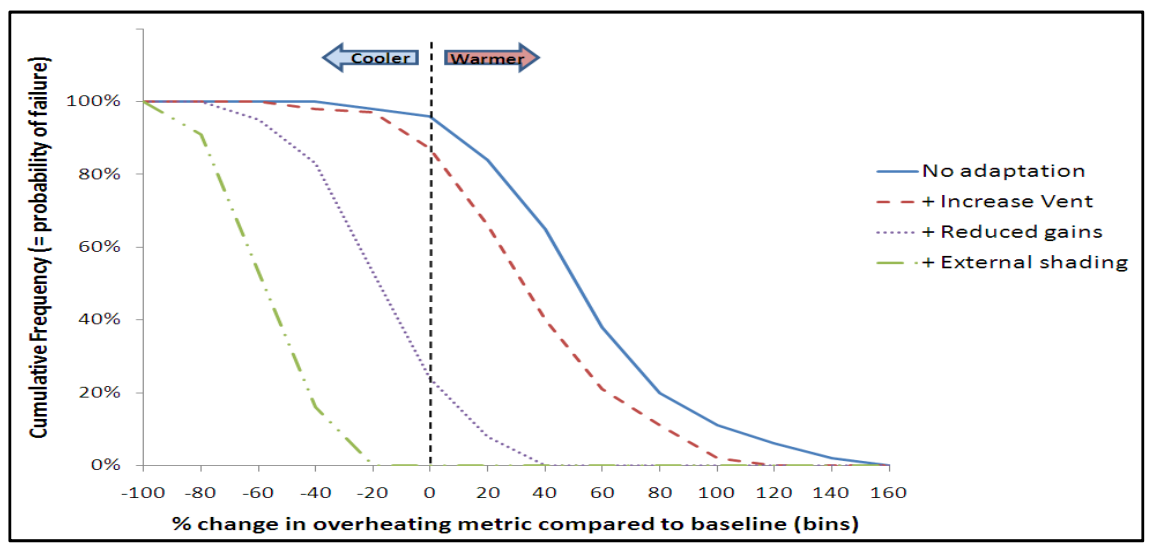

Figure 1: Probabilistic heating curve for a future climate scenario.

\section{Methodology}

A specific workshop was held in London in September 2013. The target audience selected included experienced building design professionals as well as local authorities and housing associations, managers within architectural practices and building services engineering consultancies, and experienced members of corporate organisations. This was to ensure the participants were in a position to facilitate adoption of the work by their organisations with the ultimate effects of (i) significantly reducing time for evaluating overheating risk of buildings at design stage, and (ii) increasing confidence of clients in the resilience of the design proposals to future climate change. Contact was made with 98 interested parties leading to 35 attendees: $34 \%$ from consultancy firms, 29\% other academics, $9 \%$ local authority, 9\% independent industry body, $6 \%$ housing association, $6 \%$ architects, $3 \%$ housing developers and $6 \%$ other (retired or unknown).

\subsection{Presentations}

The workshop comprised two sessions, firstly the research was explained in a series of presentations across the afternoon. Different aspects of the project such as, overheating in buildings, mitigation and adaptation of climate change in buildings, probabilistic future climates, LCF tool development, engagement with industry, were explained during these presentations.

\subsection{Discussion session}

Secondly, a discussion session asked attendees to work together to provide clientbased solutions to the issue of how to represent overheating risk. The attendees were split into four potential 'client types', based on the individual's areas of expertise requested at the time of registering attendance. The discussion required the attendees to work in groups acting on behalf of their particular client type. With eight groups in total, each client type had two groups taking part. The groups 
were to imagine an overheating assessment had been carried out, and that there were six potential output styles available to use as explained below.

\subsubsection{Clients}

Local Authority (LA) - has ISO 14001 [13] status and is attempting to be the most sustainable council in its region. They are looking to assess their building stock, starting with schools - both the existing buildings and any proposed new schools. This will incorporate energy assessment, Building Research Establishment Environmental Assessment Method (BREEAM) [14] assessment, and an overheating assessment. The client would like an assessment of the risk of future and current overheating in its schools. It must be done within a tight budget, but the client must be confident of the findings.

Housing Association (HA) - has a stock of 60,000 homes across London and the South East. The stock is quite varied, ranging from older solid-walled dwellings to more recent Code for Sustainable Homes 4 and 5 [15]. Client is building capacity for 300 new leases in London, and wants to ensure that they will not overheat now, or at any point in the lifespan of the homes (up to 2080). They need a report that indicates the likelihood of particular building designs overheating, which is easily replicable on their website and in company reports.

Small-Medium Enterprise (SME) - is expanding due to success and is moving into new offices. The new offices have already caused some discomfort to previous occupants in the summer and there is concern that there may be further overheating in the future. There is the possibility of the analysis being carried out on further buildings in the future, but the current concern is with just a single office building. They are looking into adaptation options for the office, but need them to be low carbon to fit with the objectives of the company.

Corporate (CE) - is a large, multinational corporation and has ISO 14001 [13] accreditation. The client has a mixed building stock of office, retail and factory units though would like a detailed analysis on a small number of buildings to guide future decisions on Heating Ventilation and Air Conditioning technologies and general building design. This will also aid them in identifying priority buildings that should be refurbished for adaptation to a warmer climate.

\subsubsection{Output styles}

For each output type the attendees suggested advantages and disadvantages, and identified a preferred output display. This information was collated so each client had four preferred output styles, coming from the two groups. The five main output types are described in Figure 2. The attendees were additionally invited to highlight a sixth option of their own design if the pre-determined five options were not suitable.

\section{Results and discussion}

From the groups' responses it was possible to determine reasons for the preferred choices, as well as the advantages and disadvantages of using each style for each client. What follows is a review of the output styles for each client type (where quotation marks are used it signifies a comment made by an attendee). 
(a) LCF probabilistic graph

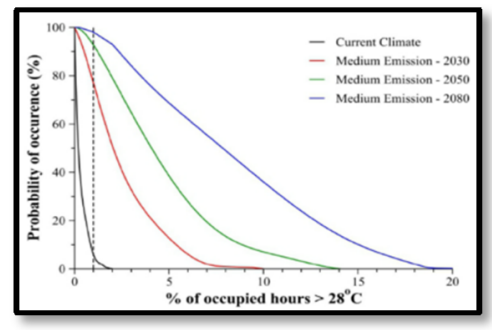

(b) LCF risk matrix

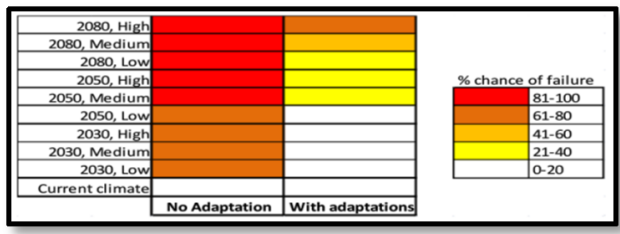

(c) Bar chart

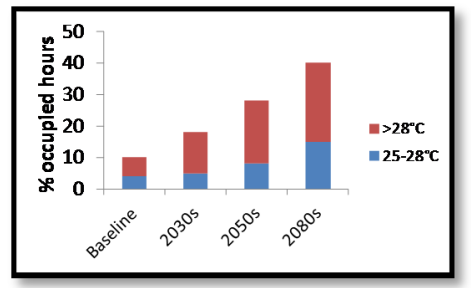

(d) "EPC-style" grades

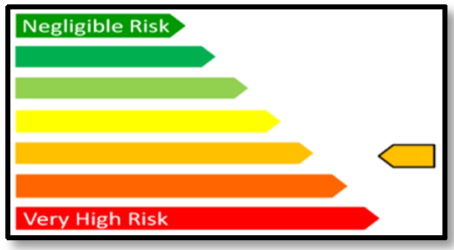

\section{(e) Qualitative descriptors}

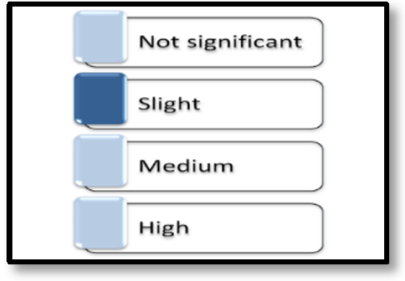

- Full spectrum of risk calculated by the LCF tool based on a chosen failure criteria

- Based on UKCP 2009

- Shows several future climate scenarios at the same time, though a second graph would be required to show any adaptation scenarios

- Allows the client to compare failure criteria on the same graph (e.g. $2 \%$ of hours above $28^{\circ} \mathrm{C}$ rather than $1 \%$ )

- Requires dynamic simulation of building(s)

- Simple colour-coded matrix based on the LCF tool (using UKCP 2009)

- Allows client to compare multiple future climate scenarios at the same time

- Shows pre and post-adaptation results

- Requires dynamic simulation of building(s)

- Simple bar chart for chosen temperature ranges

- Non-probabilistic (based on, for example, UKCIP02 [16])

- Based on the familiar UK Energy Performance Certificate (EPC)-style rainbow rating [17], with the arrow denoting where on the scale the building sits

- More suitable for a single building and climate scenario

- Less amenable for use with UKCP 2009 directly

- Based on any form of building modelling

- Can be linked to, for example, steadystate Standard Assessment Procedure (SAP) and Simplified Building Energy Mode (SBEM) assessments of overheating

- Likely to require simple, nonprobabilistic climate information (i.e. less suitable for UKCP 2009)

- More suitable for a single future climate scenario, perhaps linked with energy performance assessment

Figure 2: Overheating analysis output types suggested. 


\subsection{Local authority (LA)}

The LCF probabilistic graph was very "comprehensive" and could identify different failure thresholds in different stock. However, it was suggested it might be too complicated to use for an LA. Additionally, the cost to calculate such an output was felt to be prohibitive. The LCF risk matrix was seen to be useful for decision making with respect to staged retrofit plans, and the relationship between risk and timeline was clear. The information was felt to be displayed in a simple way, and the comparison with the "business as usual" was found to be "very useful". However, it was felt the chance of failure could have been clearer. The Bar Chart was a familiar output style for reporting, but one that could provide more information than was originally being suggested; e.g., adding an overheating threshold line. The simplicity of the image makes the EPC-style grading suitable for display purposes, although it is not useful for most decision making as it only contains a single output, does not contain threshold information, nor suggest what could be done to achieve an acceptable standard. The simplicity may be a disadvantage when assessing a complex building such as a school. If this grading were to be used, it was suggested that more detailed labelling of each grade be used or fewer grades be used. The Qualitative Descriptors (QD) were useful for display for a non-technical audience, and could highlight the degree of urgency with which action needs to be taken, however the simplicity was seen as the main disadvantage as it lacks definition. The inability to include probabilistic outcomes was also given as a disadvantage, in addition to not being conducive to decision making, understanding or contributing to a cost-benefit analysis. One alternative was suggested (Figure 3 ) for display purposes. However it could require complex calculations and could result in too much information for a display, depending how many scenarios are calculated because each would require a 'With adaptation' and 'Without adaptation' label:

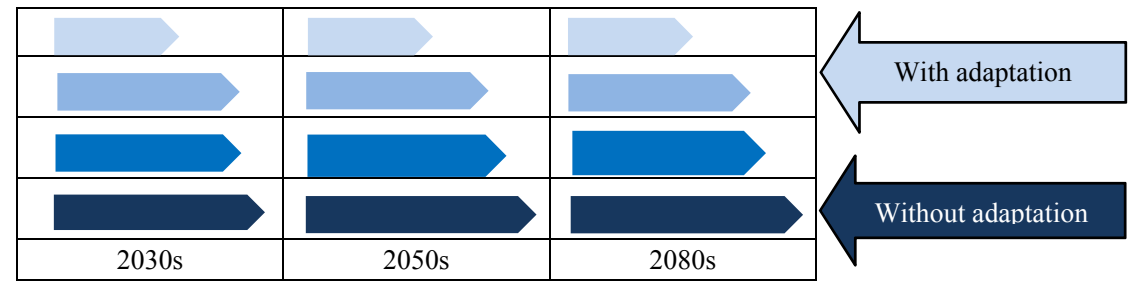

Figure 3: Suggested overheating risk assessment output type for an LA.

\subsection{Small-medium size enterprises (SMEs)}

The LCF probabilistic graph appeared to provide an SME client with a good level of data, giving the whole picture in one image, though it was felt that explanation is needed to accompany the graphical display, and that the complexity may imply greater accuracy than is actually achieved. The LCF risk matrix was straightforward and clear to understand, with the inclusion of the current situation and the difference with adaptations being of benefit. Additionally, it was suggested 
that the graphic could be expanded for different adaptation types or sequential adaptation. The Bar Chart was also seen to be a clear and straightforward graphic, providing the timeline and showing the different thresholds so a client can choose a forward outlook time period. However, the adaptation results need to be added, and a target should be added, with care given over using a stacked graph for ease of interpreting. The use of an EPC-style grading graphical display familiar and well known, but could also be confused with an EPC, therefore it could be interpreted as including energy performance in some way. This particular display lacks the temporal feedback that the others provide, and it is not clear what the 'average' is, nor where a building should be. It was also described as "overly simplistic". The $Q D$ were not appreciated due to their simplistic nature, lack of information, and in one case were described as "not scary enough". An alternative graphic was suggested (Figure 4), including embodied and operational carbon results, which includes primary colours rather than words, to remove any linguistic barriers.

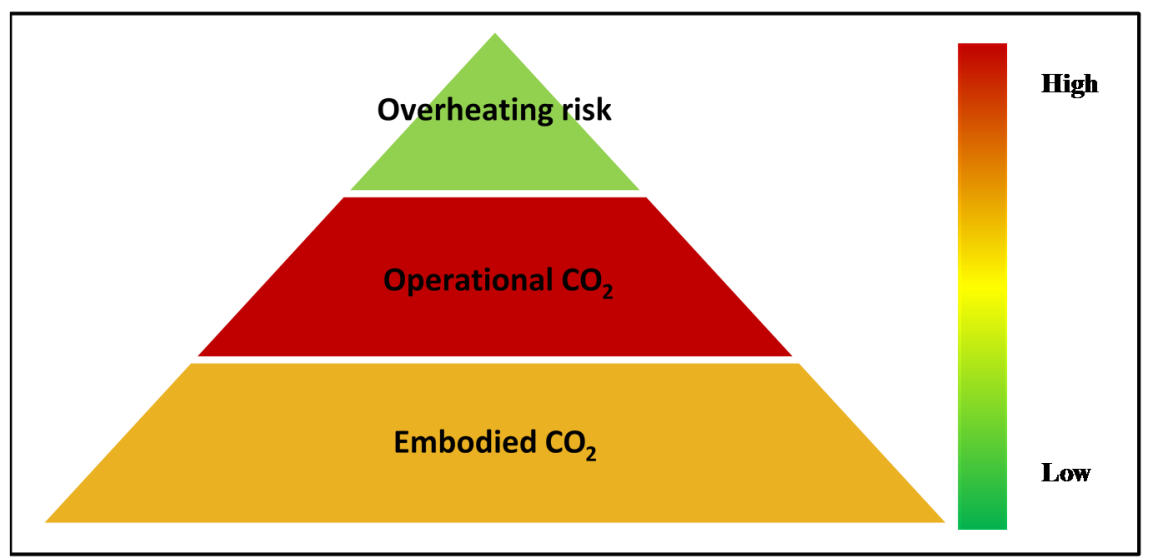

Figure 4: $\quad$ Suggested overheating risk assessment output type for an SME.

\subsection{Housing association (HA)}

The LCF probabilistic graph has benefits for decision making in future management, pre-build use, and the capability to add an easy-to-understand visual display of the effect of making adaptations. However, it does need explanation, and its use for a non-technical audience is doubted. The Risk Matrix is useful for those in the industry who respond to risk, such as financial professionals, and enables the user to relate the results to cost benefit analysis early in the design process, primarily for the existing building stock. However, for a HA, it was felt that there needs to be a way to identify the different dwellings within their stock. The Bar Chart's inclusion of a baseline was beneficial, as was the use of red for the higher risk situation of temperatures over $28^{\circ} \mathrm{C}$. However, it was felt the graph was too simplistic for decision making, and also for a whole Association portfolio, and would have to be used for a single building. The EPC-style grading was 
instantly recognisable and people can immediately relate to it. However, it could be easy to misinterpret as it is so similar to the EPC, and would only be the average for the whole stock unless done individually. The $Q D$ were good for easy communication to tenants, however, it is also simplistic and not immediately clear. An alternative, below, was suggested in the form of using the LCF risk matrix colour on a site plan of the housing stock, with different maps for different adaptations.

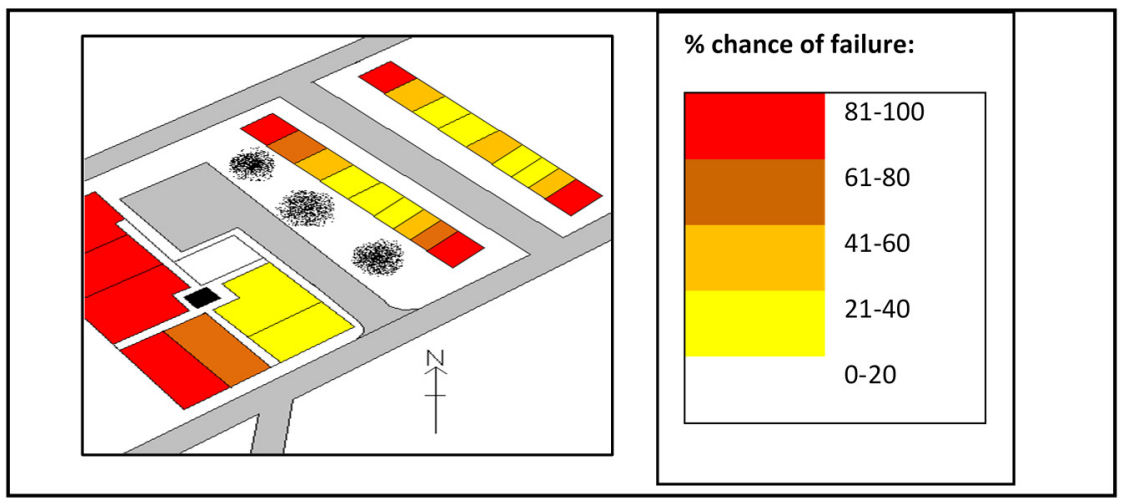

Figure 5: Suggested overheating risk assessment output type for a HA.

\subsection{Corporate (CE)}

The $L C F$ probabilistic graph gives very good feel for the sensitivities in the calculation, and is good for a technical level of understanding. However, simplification would be needed for reporting to senior management such as CEOs or Board members, and multiple diagrams would be needed to show the effect of adaptations. The $L C F$ risk matrix facilitates an understanding of complex information, including scenarios and adaptations, and is good for decision making. However, the matrix doesn't include financial and procurement risk, only overheating, therefore the graphical display could be open to misinterpretation. Additionally, the colours should be reconsidered, with one suggestion being green to red, another being white to green. It was suggested the colours should be more emotive. The Bar Chart gives a good feel for the changes occurring in the building over time, and is a familiar style of display to professionals, with the ability to display multiple scenarios. However, a threshold needs to be displayed for the chart to be useful for decision making, it is not intuitively understandable to the general public, and it does not include the effect of any adaptations. Additionally, similarly to the LCF risk matrix, the colours should be reconsidered, as red and blue suggest hot and cold, rather than hot and slightly less hot. The benefits of using an EPC-style grading system in the CE sector included the familiarity within the general public. Additionally, the use of red to green provides "emotional engagement" as red is traditionally bad and green traditionally good. The disadvantages of using such a display are that it doesn't provide numbers, whether 
energy, temperature or financial - although these could be added. The $Q D$ were "low impact", "less intelligible" than other displays, lacking clarity and "too woolly". There was felt to be scope for extending the grading system (Figure 6) to show the current situation as well as the situation in years to come, for example:

\section{Negligible Risk}

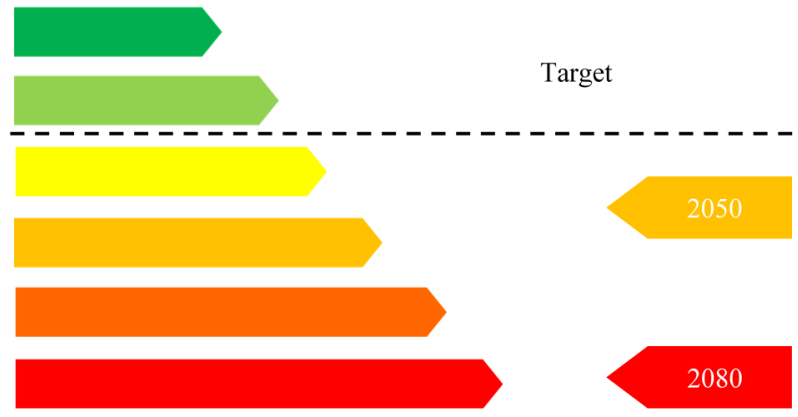

Figure 6: Suggested overheating risk assessment output type for a CE.

\subsection{Preferred choices}

Further to the discussion of each output type, the groups were asked to rank preferred outputs for their particular client. These are shown in Figure 7. Each group (with two groups per client type) had a first and second choice, giving four choices for each client type (each row), except for the "CE" client, which is

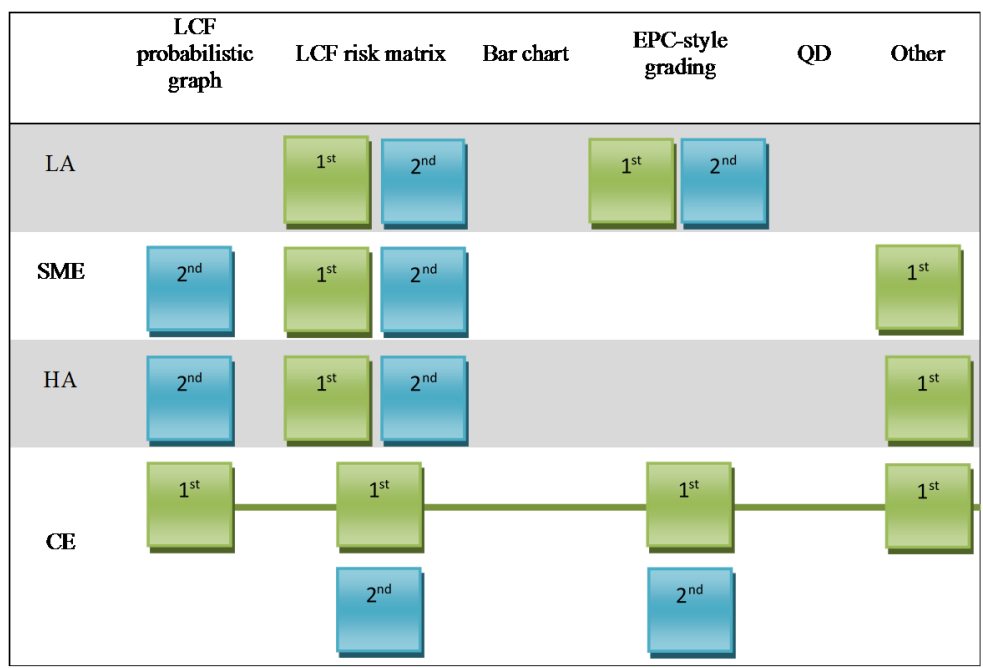

Figure 7: Preferred outputs for clients. 
explained further below. For the $L A$, the preferred output display was the risk matrix for decision making, ease of understanding, staged retrofit potential, and ability to incorporate adaptations. Equally, the EPC-style grading was preferred for the ease of display and fitting in with limited LA budgets. For SMEs, the groups liked the risk matrix for its clear communication, and how it identifies where adaptations push failure further into the future. It is also a familiar style but more comprehensive than EPC grading. The probabilistic graph was selected to promote in-depth modelling, as it was felt that in-depth modelling should be done irrespective of cost. The 'Other' option chosen was that of the pyramid displaying embodied and operational carbon as well as overheating risk.

The same output display options were chosen by those representing $H A$. The risk matrix was preferred for estate agents and for existing stock, while the probabilistic graph was preferred for new-build stock. The 'Other' option preferred was the amended risk matrix on a site plan, which could include financial aspects for a cost benefit analysis. The bar chart, EPC-style grading and qualitative descriptors were all too simple as they assumed identical stock. The groups discussing preferred outputs for the $C E$ sector were unresolved on the 'best', as each has merit depending on the audience. For technical and professional analysis, the probabilistic graph was preferred. For decision making, the risk matrix was preferred. For communication purposes, the EPC-style grading was preferred and finally, the 'Other' option of the amended EPC-style grading was preferred as it could combine with financial risk.

\section{Conclusions}

This paper has presented the results of a workshop hosted by the LCF research team to disseminate the outcomes of the LCF-tool, as well as obtaining some feedback on the use and applicability of its outcomes. The workshop was held in London with a participation of around 35 industry professionals. The general consensus suggests the LCF tool would benefit from having a simpler form of output alongside a more complex research output.

The qualitative descriptors were universally not selected as preferred outputs as they are too simplistic and do not contain enough information. For LA type clients, a comprehensive probabilistic style output was seen to be too complicated and too expensive to be widely adopted. An EPC style display was thought to be immediately understandable, and perhaps a hybrid output based on this would be most useful. For SMEs a matrix style of output was preferred, and was considered to offer the greatest flexibility in presentation of results and recommendations. An EPC, while easily understood, was felt not to offer sufficient information. For HA clients, a preference for avoidance of oversimplistic outputs was expressed. A matrix style output was preferred, but which could be adapted to reflect the multi-style dwelling/buildings that can coexist within a single development. A map style matrix showing risk for various buildings within a development was suggested. For a CE client, the comprehensive nature of the probabilistic graph was thought to have the most use, but it was felt that communication of the output would require simplification for explaining to 
corporate senior managers. A suggestion to be able to integrate the output with procurement and financial information was made. Overall a matrix style output was perceived to be optimal across all client types.

\section{References}

[1] IPCC. 2012. Special Reports (Online content) Available at: http://www.ipcc.ch/ipccreports/sres/emission/index.php?idp=50 (Last accessed September 2013).

[2] UK Climate Projections. 2012. (Online Content) Available at: http://ukclimateprojections.defra.gov.uk/21708 (Last accessed September 2013).

[3] Morton, T. A., Bretschneider, P., Coley, D. and Kershaw, T. 2011. Building a better future: An exploration of beliefs about climate change and perceived need for adaptation within the building industry. Building and Environment 46, 1151-1158.

[4] Defra. 2013. Bridging the gap between climate change research and practice. Available at: http://sd.defra.gov.uk/2012/02/bridging-the-gapbetween-climate-change-research-and-practice/ (Last accessed September 2013).

[5] Jenkins, D.P., Patidar, S., Banfill, P., F., G., and Gibson, G. 2011. Probabilistic climate projections with dynamic building simulation: predicting overheating in dwellings, Energy and Buildings 43, 1723-1731.

[6] Patidar, S., Jenkins, D., P., Gibson, G., and Banfill, P., F., G. 2011. Statistical techniques to emulate dynamic building simulations for overheating analyses in future probabilistic climates, Journal of Building Performance Simulation, 1940-1493, DOI: 10.1080/19401493. 2010.531144.

[7] ARCC. 2011. Adaptation and Resilience in a Changing Climate (Online content) Available at: http://www.ukcip-arcc.org.uk/ (Last accessed September 2011).

[8] Gul, M. and Menzies, G.F. 2012. Designing domestic buildings for future summers: attitudes and opinions of building professionals. Energy Policy, 45, pp. 752-761.

[9] Gul, M. and Menzies, G.F. 2011. Climate Change Projections and Office Buildings Design: A Qualitative Study. CIBSE Technical Symposium, DeMontfort University, Leicester, UK - 6th and 7th September 2011.

[10] Gul, M., Jenkins, D.P., Patidar, S., Banfill, P.F.G., Menzies, G.F. and Gibson, G. 2012. Tailoring a future overheating risk tool for existing building design practice in domestic and non-domestic Sectors. Building Services Engineering Research and Technology 33 (1), pp. 105-117.14.

[11] Jenkins, D. P., Gul, M., Patidar, S., Banfill, P.F.G., Gibson, G. and Menzies, G. 2012. Designing a methodology for integrating industry practice into a probabilistic overheating tool for future building performance. Energy and Buildings 54 pp. $73-80$. 
[12] Banfill, P.F.G., Jenkins, D. P., Patidar, S., Gul, M., Menzies, G. and Gibson, G. 2012 Towards an overheating risk tool for building design. Structural Survey 31 (4) pp. 253-266.

[13] Sheldon, C. 1997. ISO 14001 and beyond, Environmental management systems in the real world. Greenleaf publishing, Sheffield, UK.

[14] BRE Global 2014. http://www.breeam.org/ (Last accessed Jan 2015).

[15] Code for Sustainable Homes. 2010. Department for Communities and Local Government. Available at: http://www.planningportal.gov.uk/uploads/ code_for_sustainable_homes_techguide.pdf (Last accessed September 2012).

[16] UKCIP 2012. http://www.ukcip.org.uk/wizard/ (Last accessed September 2012).

[17] DECC 2014 https://www.gov.uk/government/policies/improving-theenergy-efficiency-of-buildings-and-using-planning-to-protect-the environment/supporting-pages/energy-performance-of-buildings. (Last accessed 15th Jan 2014). 